\title{
Young women with breast cancer: how many are actually candidates for fertility preservation?
}

\author{
Rebecca Moffat • Christian de Geyter • Mary E. Myrick • Seraina M. Schmid • \\ Christina Sattmann · Sibil Tschudin • Nerbil Kilic • Uwe Güth
}

Received: 8 June 2012/Accepted: 2 July 2012/Published online: 21 July 2012

(C) Springer-Verlag 2012

\begin{abstract}
Purpose There are no data regarding the actual need for fertility preservation (FP) in breast cancer (BC) patients. Our study provides a practical needs assessment for reproductive medicine by analyzing an unselected cohort of young BC patients. This assessment considers oncological factors as well as the patient's obstetrical and gynecological history and reproductive outcome after BC diagnosis. We aimed to identify how many patients are
\end{abstract}

R. Moffat · C. de Geyter

Clinic for Gynecologic Endocrinology and Reproductive Medicine, Women's Hospital, University Hospital Basel (UHB),

Spitalstrasse 21, 4031 Basel, Switzerland

R. Moffat · C. de Geyter - C. Sattmann - S. Tschudin .

N. Kilic $\cdot$ U. Güth

UHB, Breast Center, Spitalstrasse 21, 4031 Basel, Switzerland

M. E. Myrick

Department of Pediatric Surgery, Inselspital Bern,

University Hospital, 3010 Bern, Switzerland

S. M. Schmid

Spital Grabs, Frauenklinik, Spitalstrasse 44,

9472 Grabs, Switzerland

C. Sattmann · S. Tschudin · U. Güth

Division for Gynecology and Gyn. Oncology, Women's

Hospital, UHB, Spitalstrasse 21, 4031 Basel, Switzerland

N. Kilic

Department of Medical Oncology, UHB, Petersgraben 4,

4031 Basel, Switzerland

U. Güth $(\bowtie)$

Department of Gynecology and Obstetrics, Breast Center, Cantonal Hospital Winterthur, Brauerstrasse 15,

8401 Basel, Switzerland

e-mail: uwe.gueth@unibas.ch actually potential candidates for FP and how many patients might consequently use their cryopreserved gametes to achieve pregnancy.

Methods Based on a prospective BC database, we analyzed all patients who were $\leq 40$ years at initial diagnosis (time period of diagnosis: $1990-2007 ; n=100 ; 7.7 \%$ of the entire BC cohort; median age: 35.9 years).

Results Using an algorithm of exclusion criteria considering disease-specific, therapy-specific and family history characteristics, 36 patients who received chemotherapy were identified as potential "classical" candidates for FP. After 5 years, 22 women were identified as potential candidates for using their cryopreserved gametes to achieve pregnancy; the majority of these patients were childless $(n=16,72.7 \%)$ and in their late reproductive years $(n=12,54.5 \%)$.

Conclusions Our study demonstrates that in a cohort of young BC patients only a minority of women are candidates for FP. Young BC patients who wish to have children in the future usually carry risk factors both from oncological and reproductive medicine perspective. Due to this high-risk profile, the rarity of $\mathrm{BC}$ in young age and the limited number of patients who might actually have opted for FP, these women must be offered timely and multidisciplinary counseling in highly specialized centers.

Keywords Breast cancer - Fertility preservation . Oncofertility · Chemotherapy · Endocrine therapy

\section{Introduction}

In 2012, an estimated 1 in 200 women under the age of 40 will develop invasive breast cancer (BC). Invasive $\mathrm{BC}$ is the most frequent cancer of young women and the leading cancer 
causing death in the age group between 20 and 39 years [1]. Women under 40 comprise about $5 \%$ of the overall BC population [2]; many of them have not completed childbearing at the time of $\mathrm{BC}$ diagnosis. In 2009, birth rates declined for all women in the age groups from 15 to 39 years, but continued to rise for women aged 40-44 and remained unchanged among women aged 45 and older [3]. Considering the trend toward postponing child bearing to the late reproductive years, the number of childless women at diagnosis of BC will continue to increase. In a recent US survey $77 \%$ of childless women intended to have a child in the future [4]. In another survey, $75 \%$ of young cancer survivors without children stated they wanted to have children in the future and almost a third of the survivors who already had at least one child wanted to have another child [5].

Mortality rates for BC patients are decreasing but BC treatment will render many of the affected women infertile. Particularly women without children are distressed about their impaired family planning perspectives [6]. Therefore, fertility after BC treatment and preservation of fertility at diagnosis are issues that are currently receiving significant attention. The American Society of Clinical Oncology (ASCO) and the American Society for Reproductive Medicine (ASRM) have recommended that the impact of cancer treatments on fertility should be addressed with all cancer patients of reproductive age and that options for fertility preservation (FP), such as embryo cryopreservation, should be discussed routinely [7, 8]. Large cancer centers have followed these recommendations and today many cancer patients have access to reproductive medicine. According to registries of fertility centers performing FP consultations and treatments in Europe and the US, the largest group of young women counseled for cancer therapy-related FP were patients with BC, followed by women diagnosed with a lymphoma [9]. There are, however, no data regarding the actual need for FP consultations and treatments in young $\mathrm{BC}$ patients. To our knowledge, this study is the first to provide an assessment of the practical necessities in family planning and FP by analyzing an unselected cohort of young BC patients. This assessment considers oncological factors such as disease stage, oncological therapies and outcome as well as the patient's obstetrical and gynecological history and reproductive outcome after BC diagnosis. The aim of this study was to identify how many patients are actually potential candidates for FP and how many patients might consequently use their cryopreserved gametes to achieve pregnancy.

\section{Patients and methods}

The prospective relational web-based Basel Breast Cancer Database (BBCD) includes all newly diagnosed primary invasive BC cases treated at the University Women's Hospital Basel, Switzerland, since 1990. This institution comprises the largest breast center in the canton of Basel and represents the population of the region. In the BBCD disease-specific clinical, histo- and pathomorphologic features and treatment characteristics are collected. It also includes data regarding personal and family history and outcome. The data were recorded continuously from the medical files.

A standard patient history included the basic obstetrical information (such as parity, number and age of delivered children, age at live births, etc.) and gynecological data (such as, age at menarche, time of the last menstruation for pre-/perimenopausal women, menopausal state and age at last menstruation for postmenopausal women). Furthermore, a history of hysterectomy, bilateral oophorectomy, endocrine treatment (e.g., hormonal replacement therapy, use of contraceptives and other systemic or local hormonal treatments) and current method of contraception (including history of tubal ligation or vasectomy of the partner) was recorded.

For this study, we analyzed all patients who were diagnosed with invasive BC between 1990 and 2007 and who were 40 years or younger at initial diagnosis ( $n=100,7.7 \%$ of all patients with newly diagnosed BC in the above mentioned period). We had complete data regarding postoperative BC therapy and follow-up in 97 of these patients (three patients were lost to followup $\leq 3$ months after BC surgery). All cases were followed until death or, if they remained alive, for a maximum of 21 years. The median follow-up duration after BC diagnosis was 99 months (range $<1-252$ months). At the time of data collection in January 2012, the outcome data of patients still alive were not older than 3 months.

Patients diagnosed between 1990 and 2007 were routinely informed by the oncologist about the possible gonadotoxic effect of chemotherapy. However, similar to the internationally observed practice [10], they were rarely sent to a reproductive specialist. Before establishing ovarian stimulation protocols adding tamoxifen and later aromatase inhibitors [11] many oncologists considered conventional ovarian stimulation protocols used for in vitro fertilization treatments to be contraindicated due to hyperestrogenism, at least in patients with hormone-sensitive breast cancer.

We defined two subgroups of patients for which preservation of fertility might have come into consideration:

(a) The "classical" subgroup of patients who received chemotherapy, i.e., a cytotoxic therapy with the inherent hazard of fertility impairment or even permanent loss of ovarian function.

(b) A second subgroup of patients who received an adjuvant endocrine therapy only. To date, these 
patients have barely been considered when discussing reproductive measures in young BC patients. Endocrine therapy does not lead to immediate damage of ovarian tissue, but, since the recommended duration of this therapy is 5 years, a $\mathrm{BC}$ patient might reach her late reproductive years and face age-related decline in fertility, if she follows the valid therapy guidelines. In this case, preservation of fertility might be appropriate prior to endocrine therapy to improve the patient's chances for having a child after therapy completion.

Data collection methods and study design were approved by the local Ethical Review Board.

\section{Results}

The data on patients' age at diagnosis, disease stage, hormone receptor status, therapy and outcome are summarized in Table 1. In this particular subgroup of young BC patients (defined as $\leq 40$ years), $61 \%$ were 36 years or older at initial diagnosis. Fifty-eight patients $(59.2 \%)$ received adjuvant chemotherapy and 40 patients $(40.8 \%)$ had adjuvant endocrine therapy. Out of the 30 women $(30.6 \%)$ who had no systemic therapy, one-third $(n=10)$ refused the recommended adjuvant systemic therapy.

Forty percent of our cohort was childless at the time of BC diagnosis. Nine percent had three or more children (Table 2). The mean number of children in our study group was 1.03 per woman. Five women gave birth to a child after BC diagnosis and therapy (Table 2).

\section{Potential candidates for fertility preservation (Table 3)}

In order to assess how many $\mathrm{BC}$ patients were potential candidates for FP at time of $\mathrm{BC}$ diagnosis, we developed an algorithm using definitive exclusion criteria such as metastatic disease at diagnosis $(n=2)$ and history of hysterectomy $(n=2)$. Furthermore, 30 patients who did not receive systemic therapy $(n=30)$ were not considered as potential candidates for FP (some of these women might have been potential candidates for various gynecological/ reproductive medicine reasons; our aim, however, was to assess the number of patients who were candidates due to BC-related therapy). The potential candidates for FP were grouped according to the modality of tumor treatment.

Group A Fifty-six patients received adjuvant gonadotoxic chemotherapy. Of these women, 20 patients $(35.7 \%)$ were with the utmost probability, not candidates for FP since their gynecological/obstetrical history was highly suggestive of completed family planning (history of tubal ligation or vasectomy of the partner, $n=6$; patients who had $\geq$ three children, $n=4$; women whose youngest child was $\geq 8$ years old, $n=10$ ).

Thirty-six patients, i.e. $64.3 \%$ of the patients treated with chemotherapy, were identified as potential "classical" candidates for FP. The majority of them were childless at diagnosis $(n=23,63.9 \%)$ and 19 women $(52.8 \%)$ were in their late reproductive years, i.e. they were 36 years or older at the time of $\mathrm{BC}$ diagnosis.

In order to assess how many of the potential candidates for FP at the time of BC diagnosis might actually use their cryopreserved gametes, we analyzed the situation of each patient 5 years after BC diagnosis. Out of the 36 candidates, eight patients $(22.2 \%)$ would not have been able to use their cryopreserved gametes since they developed distant metastases after a median time of 21 months (range progression during adjuvant therapy-53 months). A further six patients $(16.7 \%)$ were highly unlikely to use their cryopreserved gametes because they chose to undergo adnexectomy for hormone-ablative BC therapy in the further course of disease.

The majority of the remaining 22 women who were finally identified as potential candidates for using their cryopreserved gametes were childless $(n=16,72.7 \%)$ and in their late reproductive years, i.e. they were already 41-45 years old at the time of potentially using the cryopreserved gametes to achieve pregnancy ( $n=12,54.5 \%$ ).

Group $B$ Out of ten patients who received endocrine therapy only, five women had hypothetic exclusion criteria for FP. From the remaining five patients, three women were identified as potential candidates for using their cryopreserved gametes 5 years after BC diagnosis.

\section{Discussion}

Women who face infertility due to oncological therapy have few options to preserve their fertility before beginning cancer treatment [12]. Currently, the most common techniques of FP are cryopreservation of embryos, oocytes and ovarian tissue. Cryopreservation of embryos is an established method, whereas cryopreservation of unfertilized oocytes and ovarian tissue are still considered experimental, because neither efficacy nor outcome has yet been established in large numbers [7]. Ovarian tissue banking is considered for younger women under the age of 35 years [13] who have no time for an ovarian stimulation which usually needs about 2-3 weeks of time. It requires one laparoscopy to retrieve the tissue and at least one other laparoscopy to autotransplant the ovarian tissue orthotopically when the woman wants to get pregnant. As of October 2011, we have knowledge of 17 children being born after autotransplanting cryopreserved ovarian tissue [14]. Apart from requiring two invasive surgical procedures, removing a whole or at least half an ovary 
Table 1 Age, disease stage, hormone receptor status, treatment and outcome characteristics of young breast cancer patients

\begin{tabular}{|c|c|}
\hline Entire cohort, number of patients & 100 \\
\hline \multicolumn{2}{|l|}{ Age } \\
\hline Mean (range) & $35.9(26-40)$ \\
\hline Age group: $26-30$ years & 10 \\
\hline Age group: $31-35$ years & 29 \\
\hline Age group: $36-40$ years & 61 \\
\hline \multicolumn{2}{|l|}{ TNM stage $^{\mathrm{a}}$} \\
\hline Stage I & 36 \\
\hline Stage II & 45 \\
\hline Stage III & 17 \\
\hline Stage IV & 2 \\
\hline \multicolumn{2}{|l|}{ Hormone receptor status } \\
\hline ER/PR positive & 51 \\
\hline ER positive/PR negative & 9 \\
\hline ER negative/PR positive & 6 \\
\hline ER/PR negative & 26 \\
\hline Not available & 8 \\
\hline \multicolumn{2}{|l|}{ Surgery } \\
\hline Breast-conserving therapy & 64 \\
\hline Mastectomy & 41 \\
\hline No surgery & 1 \\
\hline \multicolumn{2}{|c|}{ Systemic neoadjuvant/adjuvant therapy, $n=98^{\mathrm{b}}$} \\
\hline No systemic therapy (\%) & $30(30.6)$ \\
\hline Chemotherapy alone (\%) & $28(28.6)$ \\
\hline Chemotherapy + endocrine therapy (\%) & $30(30.6)$ \\
\hline Endocrine therapy alone $(\%)$ & $10(10.2)$ \\
\hline \multicolumn{2}{|l|}{ Outcome } \\
\hline Alive, no evidence of breast cancer & 57 \\
\hline Alive with metastatic breast cancer & 9 \\
\hline Died of breast cancer & 29 \\
\hline Died of other disease & 2 \\
\hline Follow-up $<3$ months & 3 \\
\hline
\end{tabular}

$E R$ estrogen receptor, $P R$ progesteron receptor

a Stage classification according to the AJCC/UICC TNM Classification, 7th edition. For 13 patients, who were treated with neoadjuvant therapy, the tumor stage after surgery (ypTNM) was considered

${ }^{\mathrm{b}}$ Exclusion of patients with stage IV disease at diagnosis $(n=2)$

will decrease the woman's ovarian reserve in any case, thus definitely lowering her chances for spontaneous pregnancy and increasing her risk for premature menopause [15].

The American Society of Clinical Oncology (ASCO) and the American Society for Reproductive Medicine (ASRM) have recommended that the impact of cancer treatments on fertility should be addressed to all cancer patients of reproductive age, and that options for FP, such as embryo cryopreservation, should be discussed routinely $[7,8]$. We agree with these recommendations and strongly
Table 2 Parity and reproductive outcome of young breast cancer patients $(n=100)$

\begin{tabular}{|c|c|}
\hline \multicolumn{2}{|l|}{ Parity at diagnosis } \\
\hline $\mathrm{P} 0$ & 40 \\
\hline P1 & 29 \\
\hline $\mathrm{P} 2$ & 22 \\
\hline P3 & 6 \\
\hline P4 & 3 \\
\hline Mean number of children & 1.03 \\
\hline Mean maternal age at birth of first child (range) & $27.9(17-37)$ \\
\hline No. of pregnancy-associated $\mathrm{BC}^{\mathrm{a}}$ & 6 \\
\hline $\begin{array}{l}\text { Patients giving birth to a child after diagnosis } \\
\text { of } \mathrm{BC}(\%)^{\mathrm{b}}\end{array}$ & $5(5.2)$ \\
\hline Spontaneous pregnancies $^{c}$ & 4 \\
\hline Pregnancy after oocytes donation & 1 \\
\hline Pregnancies after chemotherapy ${ }^{\mathrm{d}}$ & 3 \\
\hline Average age at diagnosis of $\mathrm{BC}$ (range) & $33(30-39)$ \\
\hline Average maternal age at birth of child (range) & $38.6(36-43)$ \\
\hline Months between diagnosis and birth of child (range) & $66.8(14-117)$ \\
\hline \multicolumn{2}{|l|}{$B C$ breast cancer } \\
\hline \multicolumn{2}{|c|}{$\begin{array}{l}\text { a Definition: breast cancer diagnosed during pregnancy or within } 1 \text { year } \\
\text { after giving birth }\end{array}$} \\
\hline \multicolumn{2}{|c|}{$\begin{array}{l}\text { b Not considered: patients lost to follow-up after less than } 3 \text { months } \\
(n=3)\end{array}$} \\
\hline \multicolumn{2}{|c|}{$\begin{array}{l}c \text { Three pregnancies occurred after early discontinuation of endocrine } \\
\text { therapy with tamoxifen }\end{array}$} \\
\hline
\end{tabular}

support the approach that oncologists or oncological surgeons should routinely refer young BC patients to specialists for FP prior to treatment as early as possible. This study aimed to assess the number of women in an unselected cohort of young BC patients for whom such a consultation on options for FP would go beyond the means of a purely informational discussion and who actually might choose the option of FP. This needs assessment might serve as a basis for reproductive medicine centers planning FP programs for cancer patients.

In order to assess how many $\mathrm{BC}$ patients are actually candidates for FP, it is important to keep in mind that, even in the cohort of young patients, the mean age is, at least from the perspective of reproductive medicine, comparatively high. In our cohort, $61 \%$ of the patients were at least 36 years old; in comparison, the cohort of lymphoma patients who might be considered for FP is approximately 10-15 years younger [9]. This high percentage of women in their late reproductive years has two important implications for the question issued in this study:

1. Many women have completed childbearing at the time of $\mathrm{BC}$ diagnosis and the impact of oncologic therapy on fertility is secondary. 
In our study, we gave special consideration to the family structure (number and age of children) of our cohort and hypothesized that women with more than three children and women whose oldest child was already 8 years or older have most likely completed childbearing and definitely refrain from $\mathrm{FP}$ in the face of newly diagnosed $\mathrm{BC}$. Therefore, we excluded these patients as potential candidates for FP as well as women who had a history of tubal ligation or vasectomy of the partner, both highly suggestive of completion of family planning. We are aware that these factors are debatable but we are convinced that, out of the women who meet our practice-orientated exclusion criteria, only a clear minority will vote for FP, particularly in the light of the next point.

2. The live birth rate after assisted reproductive technology (ART) sharply declines with increasing maternal age.

The live birth rate in ART using freshly collected oocytes drops from $32.0 \%$ at age 36 to $18.7 \%$ at age 40 and to $2.9 \%$ at age 44 [16]. ART cycles using frozen gametes (as it would be the case after FP) show a decrease in implantation rates as maternal age increases with an implantation rate of $25 \%$ in women less than 35 years and an implantation rate of $18 \%$ for embryos of women aged 38-40 years [16]. These data were collected from a healthy population. To date, there is no evidence from controlled trials on the live birth rate of $\mathrm{BC}$ cancer patients who underwent FP. However, results from stimulation cycles in oncologic patients suggest a disease-related poorer response to ovarian stimulation [17]. We have to assume that the aforementioned ART results are considerably better than those that we can expect from cancer patients undergoing FP in an emergency situation. These low success rated could discourage patients from undergoing FP. Some patients fear that a time-consuming fertility preserving therapy might lead to a delay in treatment and that ovarian stimulation might negatively impact the prognosis of hormone-receptor positive BC [18]. Delay in treatment of patients with $\mathrm{BC}$ can be avoided by prompt referral [12]. To date, controlled ovarian stimulation in combination with aromatase inhibitors does not seem to have a negative impact on recurrence rates [19].

BC-related FP comes only into consideration for patients who receive a cytotoxic chemotherapy (the "classical" group for FP) or, up until now less common, for patients who receive a long-lasting endocrine therapy. In our cohort, however, $30 \%$ of the patients did not receive such a systemic therapy. Notably, a considerable number of the patients who refused therapy did so with the explicit wish still to have children. These patients made this decision fully conscious about the impact of their decision on the outcome of a potentially life-threatening disease. Some of these patients might have rejected systemic BC treatment for financial reasons because health insurances in Switzerland do not offer coverage for fertility preservation. This clearly highlights the enormous pressure that many young women face in this situation. The desire to achieve pregnancy despite the presence of BC might not only result in a non-compliance of therapy (i.e., not to start a recommended therapy) but also in a high rate of non-persistence to therapy. From the five patients who gave birth to a child after BC therapy in our cohort, three women discontinued ongoing endocrine therapy prematurely with the explicit intention to get pregnant. A further four patients stopped therapy for the same reason but pregnancy has not yet occurred. Several studies demonstrated that non-compliance and non-persistence was highest [20-23] in young BC patients. It was assumed that these women might not have adjusted to a diagnosis of $\mathrm{BC}$ as well as older women and therefore were also less willing to accept or more likely to experience therapy-related side effects [24, 25]. The wish for childbearing, or at least to maintain fertility, seems to be an additional factor that influences the decision not to accept a recommended oncological therapy or to discontinue therapy prematurely. Findings of a web-based survey suggest that a large proportion of women-especially those younger than 30 years-overestimate their risk of becoming postmenopausal with BC therapy. This misperception is worrisome in light of the fact that nearly one-third of respondents indicated that fertility concerns impacted on their treatment decisions [26]. Therefore, oncologists must openly discuss fertility factors and a multidisciplinary approach including reproductive specialists should be attempted to provide the patients with adequate information.

In our analysis, we identified approximately 35-40\% (the first percentage includes patients who had chemotherapy; the latter percentage also includes patients who had endocrine therapy only) of young BC patients as potential candidates for FP. From the patients who received chemotherapy, approximately $65 \%$ were assessed to be potential FP candidates. Notably, the majority of these women were in their late reproductive years. Our hypothetic analysis of the patients who were candidates for FP showed that approximately $40 \%$ of these patients no longer were candidates for using their cryopreserved gametes after 5 years, in the majority of the cases due to the development of distant metastatic disease.

We consider that our quantitative approximation of potential FP candidates based on retrospective data might be a realistic assessment of the number of women afflicted with BC aiming at FP. One might criticize that our cohort includes a lead-time bias and included a too high number of patients who did not receive systemic therapy (according to therapy guidelines in the early 1990s; approximately 
Table 3 Potential candidates for fertility preservation at the time of diagnosis $(n=100)$

\begin{tabular}{ll}
\hline Exclusion criteria for fertility preservation & 34 \\
Disease-associated criteria: metastatic disease at diagnosis & 2 \\
Patient-associated criteria: history of hysterectomy & 2 \\
Patients who had no systemic adjuvant therapy & 30 \\
Potential candidates for fertility preservation & \\
Group A ("chemotherapy") & 56 \\
Hypothetic exclusion criteria for fertility preservation: & 20 \\
History of tubal ligation or vasectomy of the partner & 6 \\
Patient with 3 or more children & 4 \\
Youngest child $\geq 8$ years & 10 \\
Remaining candidates for fertility preservation/thereof & $36 / 23$ \\
nulliparous & \\
Aged 26-30 years at diagnosis/thereof nulliparous & $6 / 5$ \\
Aged 31-35 years at diagnosis/thereof nulliparous & $11 / 6$ \\
Aged 36-40 years at diagnosis/thereof nulliparous & $19 / 12$ \\
Potential candidates for fertility preservation and further & 36 \\
events during disease course & \\
Developed metastatic disease within 2 years & 6 \\
Developed metastatic disease within 5 years & 2 \\
Underwent adnexectomy for hormone-ablative BC therapy & 6 \\
Lost to follow-up & -
\end{tabular}

Potential candidates who might use fertility reserve/thereof $\quad 22 / 16$ nulliparous

Aged 26-30 years at diagnosis/thereof nulliparous

Aged 31-35 years at diagnosis/thereof nulliparous

Aged 36-40 years at diagnosis/thereof nulliparous

Group B ("endocrine therapy only")

Hypothetic exclusion criteria for fertility preservation:

History of tubal ligation or vasectomy of the partner

Patient with 3 or more children

Youngest child $\geq 8$ years

Remaining candidates for fertility preservation/thereof nulliparous

Aged 26-30 years at diagnosis/thereof nulliparous

Aged 31-35 years at diagnosis/thereof nulliparous

Aged 36-40 years at diagnosis/thereof nulliparous

Potential candidates for fertility preservation and further

events during disease course

Developed metastatic disease within 2 years

Developed metastatic disease within 5 years

Underwent adnexectomy for hormone-ablative BC therapy 1

Lost to follow-up

Potential candidates who might use fertility reserve/there of $3 / 2$ nulliparous

Aged 31-35 years at diagnosis/thereof nulliparous

Aged 36-40 years at diagnosis/thereof nulliparous

$B C$ breast cancer

two-thirds of the patients who did not have systemic therapy had initial diagnosis between 1990 and 1995) and that there might be a higher percentage of young $\mathrm{BC}$ patients who will accept the recommended systemic therapy in the future. This, and the fact that survival rates for BC patients are increasing steadily, would lead to a higher number of potential FP candidates. On the other side, we did not consider personal motivation of the family structures of the patients of our cohort. In a group of young childless women in their mid-thirties, there might be a considerable percentage of women whose childlessness is not a result of a yet unfulfilled wish for motherhood but a conscious decision not to have children. For these women, the offer of FP is not relevant. In our assessment, these patients were not excluded from the number of potential FP candidates. We feel that both factors would neutralize each other enough that our assumed number of potential FP candidates would still be a realistic assessment for the future.

One major problem of FP preservation is the considerable time pressure on patients and their physicians [27]. It would be essential to identify candidates for FP at an early point so that patients are able to make their decisions both on oncological therapy and FP [28, 29]. Information transfer is challenging in this ethically and emotionally complex situation [30] preferably with a fertility specialist and at a time when the patient's decision is likely to impact outcome.

Biological parenthood is an important goal for most cancer survivors and the experience of cancer might well add to the appreciation of parenthood [5]. We think that young women who had FP benefit emotionally from the enhanced hope of future motherhood. On the other side, the optimistic prospect of future parenthood must not detract from the real hazards of BC. In our study cohort, $36 \%$ developed distant metastatic disease. From the 36 patients who received chemotherapy and were identified as potential candidates for FP, six patients had early progression within 2 years and three further patients developed distant metastases in the further course of disease.

Our study demonstrates that in a cohort of young BC patients only a minority of women are candidates for FP. Young $\mathrm{BC}$ patients who wish to have children in the future usually carry risk factors both from oncological and reproductive medicine perspective. Due to this high-risk profile, the rarity of $\mathrm{BC}$ in young age and the limited number of patients who might actually chose FP, these women must be offered timely and multidisciplinary counseling in highly specialized centers. To improve our knowledge and understanding of the challenging situation of young $\mathrm{BC}$ patients with the wish to have children in the future, the data of these patients must be collected in large multicenter databases (such as FertiPROTECT, FertiSave). Ongoing studies and those currently being designed will "study the different features contributing to the "puzzle" of safe and successful pregnancy after BC" [31]. 
Conflict of interest The authors declare that there are no financial or personal relationships with other people or organizations that could inappropriately influence the work reported or the conclusions, implications, or opinions stated.

\section{References}

1. Siegel R, Naishadham D, Jemal A (2012) Cancer statistics, 2012. CA Cancer J Clin 62:10-29

2. Bland KI, Menck HR, Scott-Conner CE et al (1998) The National Cancer Data Base 10-year survey of breast carcinoma treatment at hospitals in the United States. Cancer 83:1262-1273

3. Martin J, Hamilton B, Ventura S et al. Births: Final data for 2009. In National vital statistics reports. Hyattsville, MD: National Center for Health Statistics 2011

4. Martinez GM, Chandra A, Abma JC et al (2002) Fertility, contraception, and fatherhood: data on men and women from cycle 6 of the 2002 National Survey of Family Growth. Vital Health Stat 23:1-142

5. Schover LR, Rybicki LA, Martin BA, Bringelsen KA (1999) Having children after cancer. A pilot survey of survivors' attitudes and experiences. Cancer 86:697-709

6. Canada AL, Schover LR (2012) The psychosocial impact of interrupted childbearing in long-term female cancer survivors. Psychooncology 21:134-143

7. Ethics Committee of the American Society for Reproductive Medicine (2005) Fertility preservation and reproduction in cancer patients. Fertil Steril 83: 1622-1628

8. Lee SJ, Schover LR, Partridge AH et al (2006) American Society of Clinical Oncology recommendations on fertility preservation in cancer patients. J Clin Oncol 24:2917-2931

9. Lawrenz B, Jauckus J, Kupka MS et al (2011) Fertility preservation in $>1,000$ patients: patient's characteristics, spectrum, efficacy and risks of applied preservation techniques. Arch Gynecol Obstet 283:651-656

10. Forman EJ, Anders CK, Behera MA (2010) A nationwide survey of oncologists regarding treatment-related infertility and fertility preservation in female cancer patients. Fertil Steril 94:1652-1656

11. Oktay K, Buyuk E, Davis O et al (2003) Fertility preservation in breast cancer patients: IVF and embryo cryopreservation after ovarian stimulation with tamoxifen. Human Reprod 18:90-95

12. Sonmezer M, Oktay K (2006) Fertility preservation in young women undergoing breast cancer therapy. Oncologist 11:422-434

13. von Wolff M, Donnez J, Hovatta O et al (2009) Cryopreservation and autotransplantation of human ovarian tissue prior to cytotoxic therapy-a technique in its infancy but already successful in fertility preservation. Eur J Cancer 45:1547-1553

14. Dittrich R, Lotz L, Keck G et al (2012) Live birth after ovarian tissue autotransplantation following overnight transportation before cryopreservation. Fertil Steril 97:387-390

15. de Ziegler D, Streuli I, Vasilopoulos I et al (2010) Cancer and fecundity issues mandate a multidisciplinary approach. Fertil Steril 93:691-696

16. Centers for Disease Control and Prevention, American Society for Reproductive Medicine, Society for Assisted Reproductive
Technology (2011) 2009 Assisted Reproductive Technology Success Rates: National Summary and Fertility Clinic Reports. Atlanta: US Department of Health and Human Services. Available at: http://www.cdc.gov/art/ART2009/PDF/ART_2009_Full. pdf. Accessed 21 March, 2012

17. Friedler S, Koc O, Gidoni Y et al (2012) Ovarian response to stimulation for fertility preservation in women with malignant disease: a systematic review and meta-analysis. Fertil Steril 97: $125-133$

18. Tschudin S, Bunting L, Abraham J et al (2010) Correlates of fertility issues in an internet survey of cancer survivors. J Psychosom Obstet Gynaecol 31:150-157

19. Azim A, Costantini-Ferrando M, Oktay K (2008) Safety of fertility preservation by ovarian stimulation with letrozole and gonadotropins in patients with breast cancer: a prospective controlled study. J Clin Oncol 26:2630-2635

20. Barron TI, Connolly R, Bennett K et al (2007) Early discontinuation of tamoxifen: a lesson for oncologists. Cancer 109: 832-839

21. Guth U, Myrick ME, Kilic N et al (2012) Compliance and persistence of endocrine adjuvant breast cancer therapy. Breast Cancer Res Treat 131:491-499

22. Hershman DL, Kushi LH, Shao T et al (2010) Early discontinuation and nonadherence to adjuvant hormonal therapy in a cohort of 8,769 early-stage breast cancer patients. J Clin Oncol 28:4120-4128

23. Partridge AH, LaFountain A, Mayer E et al (2008) Adherence to initial adjuvant anastrozole therapy among women with earlystage breast cancer. J Clin Oncol 26:556-562

24. Compas BE, Stoll MF, Thomsen AH et al (1999) Adjustment to breast cancer: age-related differences in coping and emotional distress. Breast Cancer Res Treat 54:195-203

25. Ganz PA, Schag CC, Heinrich RL (1985) The psychosocial impact of cancer on the elderly: a comparison with younger patients. J Am Geriatr Soc 33:429-435

26. Partridge AH, Gelber S, Peppercorn J et al (2004) Web-based survey of fertility issues in young women with breast cancer. J Clin Oncol 22:4174-4183

27. Kim J, Oktay K, Gracia C et al (2012) Which patients pursue fertility preservation treatments? A multicenter analysis of the predictors of fertility preservation in women with breast cancer. Fertil Steril 97:671-676

28. Lee S, Ozkavukcu S, Heytens E et al (2010) Value of early referral to fertility preservation in young women with breast cancer. J Clin Oncol 28:4683-4686

29. Thewes B, Meiser B, Rickard J, Friedlander M (2003) The fertility- and menopause-related information needs of younger women with a diagnosis of breast cancer: a qualitative study. Psychooncology 12:500-511

30. Tschudin S, Bitzer J (2009) Psychological aspects of fertility preservation in men and women affected by cancer and other lifethreatening diseases. Hum Reprod Update 15:587-597

31. Pagani O, Partridge A, Korde L et al (2011) Pregnancy after breast cancer: if you wish, ma'am. Breast Cancer Res Treat 129: 309-317 